\section{Christoph Bachmann}

\section{Curcumin}

Curcumin ist der wichtigste Inhaltsstoff von C. longa L. (Ingwergewächse), der Gelbwurz, auch Kurkuma genannt. Gelbwurzpulver enthält 5\% Curcuma, ist intensiv gelb und wird aus dem getrockneten Wurzelstock gewonnen. Als Bestandteil von Curry gibt es dem Gewürz die charakteristische gelbe Farbe.

Curcuma wird schon seit Langem in der Ayurvedischen Medizin und in der traditionellen indischen Medizin zur Entspannung und Reinigung verwendet.

\section{Pharmakologische Wirkungen}

Die entzündungshemmenden Eigenschaften von Curcumin sind seit Jahrhunderten bekannt. Csaki et al. [2] stellten folgenden Wirkungsmechanismus vor: Curcumin hemmt die von Interleukin-1 $\beta$ (IL-1 $\beta$ ) ausgelöste Bildung und die Ausschüttung von NFKappa-B sowie, daraus folgend, die von NF-Kappa-B bewirkte Bildung der proinflammatorischen Gene COX-2 und VEGF. In einer Studie mit Chondrozyten konnte die Fähigkeit von Curcumin, den Transkriptionsfaktor AP-1 zu hemmen und die durch IL-1 $\beta$ stimulierte Bildung von Nitritoxid (NO) und Prostaglandin $\mathrm{E}_{2}\left(\mathrm{PGE}_{2}\right)$ zu vermindern, belegt werden [3].

\section{Aus der Forschung}

Schweiz Z Ganzheitsmed 2016;28:321-323

Published online: November 11, 2016

\title{
Curcuma bei Arthrose und rheumatoider Arthritis: Klinische Studien dokumentieren die Wirksamkeit von Gelbwurz-Extrakten
}

Über die neuroprotektive Wirksamkeit von Curcumin, des Hauptinhaltsstoffes von Curcuma longa L., wurde bereits in der letzten Ausgabe dieser Zeitschrift berichtet [1]. Im Folgenden werden weitere klinische Studien vorgestellt, die eine zusätzliche Wirkung von Curcuma dokumentieren.

\section{Klinische Wirksamkeit}

In der Folge werden mehrere klinische Studien vorgestellt, die sich mit der Wirkung von Curcumin bzw. Curcuma-Extrakten bei Arthrose und bei rheumatoider Arthritis befassen und seine Wirksamkeit dokumentieren.

\section{Kulkarni et al., 1991}

Kulkarni et al. [4] publizierten 1991 eine randomisierte, doppelblinde, placebokontrollierte Studie, in der $42 \mathrm{~Pa}$ tienten mit Arthrose während $1 \mathrm{Mo}-$ nat ein pflanzliches, Curcuma-reiches Präparat einnahmen. Die VerumGruppe berichtete von einer positiven Wirkung auf die Schmerzen und die Mobilität.

\section{Belcaro et al., 2010}

In einer 2010 publizierten Studie von Belcaro et al. [5] wurde die Wirksamkeit eines Komplexes von Curcumin mit Phosphatidylcholin, der die Bioverfügbarkeit von Curcumin erhöht, überprüft. Dabei erhielten 100 Patienten, die an Arthrose litten, während 8 Monaten diesen CurcuminKomplex.

Die Verum-Gruppe erhielt neben der «bestmöglichen Arthrose-Behandlung» zusätzlich täglich die Menge des Curcumin-Komplexes, die 200 mg Curcuminoiden entsprach. Die Kontrollgruppe erhielt neben der «bestmöglichen Arthrose-Behandlung» keinen Komplex.

Die Wirksamkeit wurde mit verschiedenen Standard-Zielvariablen wie WOMAC (Western Ontario and McMaster Universities Arthritis Index) sowie Entzündungsmarkern wie IL-1 $\beta$, IL-6 usw. überprüft.

Verglichen mit der Kontrollgruppe konnte in der Gruppe, die neben der konservativen Behandlung auch den Curcumin-Komplex erhielt, eine bessere Wirksamkeit beobachtet werden. Erverminderte signifikant dieSchmerzen sowie die Steifheit und verbesserte die Gelenkfunktionen. Auch soziale und psychische Parameter zeigten eine deutliche Verbesserung. Die Werte der biochemischen Marker nahmen während der Behandlung deutlich ab.

\section{Chandran und Goel, 2012}

Chandran und Goel [6] überprüften die Wirksamkeit von Curcumin bei Patienten, die an rheumatoider Arthritis litten. Hierfür wurden 45 Patienten mit einer diagnostizierten rheumatoiden Arthritis in 3 Gruppen randomisiert. Die erste Gruppe erhielt $50 \mathrm{mg}$ Diclofenac, die zweite Gruppe 500 mg Curcumin und die dritte Gruppe eine Kombination beider Wirkstoffe. Die primä-

\section{KARGER}

(C) 2016 S. Karger GmbH, Freiburg

Fax +497614520714 
re Zielvariable war die Verbesserung gemessen mit dem Disease Activity Score (DAS) 28. Sekundäre Zielvariablen waren Kriterien des American College of Rheumatology (ACR), welche die Drucktoleranz und die Schwellung von Gelenken beinhalteten.

Alle 3 Patientengruppen erfuhren eine signifikante Verbesserung des DAS. Interessanterweise wurde in der Curcumin-Gruppe die grösste Verbesserung des DAS und des ACRScore ermittelt. Diese Verbesserung erreichte gegenüber der DiclofenacGruppe sogar Signifikanz. Ausserdem erwies sich die Behandlung mit Curcumin als sicher und wurde mit keinerlei Nebenwirkungen in Zusammenhang gebracht.

\section{Madhu et al., 2013}

Ein Jahr nach der Studie von Chandran und Goel [6] wurde die Studie von Madhu et al. [7] publiziert, welche die Wirksamkeit eines Curcuma-Extraktes bei Kniearthrose untersuchte. In diese randomisierte, einzelblinde, placebokontrollierte Studie wurden 120 Patienten (37 Männer, 83 Frauen) aufgenommen, die an einer Arthrose am Knie litten. Diese Patienten erhielten je zweimal täglich entweder

- 400 mg Placebo oder

- 500 mg der Polysaccharid-Fraktion von C. longa (NR-INF-02) oder

- 750 mg Glucosaminsulfat (GS) oder

- GS und NR-INF-02 zusammen.

Die Studie dauerte 42 Tage. Die Wirksamkeit der Behandlung wurde an den Tagen 21 und 42 ermittelt. Als primäre Zielvariablen wurden die Abnahme der Beschwerden im betroffenen Knie sowie dessen Funktionsfähigkeit ermittelt. Die Schmerzverminderung wurde mit der Visuellen Analogskala (VAS) und dem WOMAC-Index gemessen. Die Funktionsfähigkeit des Knies wurde von einem Orthopäden überprüft, der dazu die
Clinical Global Impressions (CGI) Scale verwendete. Die Wirksamkeit von NR-INF-02 gegenüber Placebo erwies sich in allen ermittelten Parametern als signifikant besser $(\mathrm{p}<0,05)$.

Die Patienten der Curcuma-Gruppe mussten verglichen mit der Placebo-Gruppe signifikant weniger Notfallmedikamente verwenden ( $\mathrm{p}<$ $0,01)$. Auch die klinische und subjektive Verbesserung verglichen mit Placebo entsprach diesem Bild.

Die Verträglichkeit und Akzeptanz von NR-INF-02 war sehr gut.

\section{Kuptniratsaikul et al., 2014}

Als vorläufig letzte Publikation wurde 2014 eine Studie eines thailändischen Forscherteams um Kuptniratsaikul publiziert [8]. In diese Studie wurden 367 Patienten mit einer primären Kniearthrose und einem Schmerzscore von 5 oder mehr aufgenommen. Diese Patienten wurden in 2 Gruppen randomisiert und erhielten während 28 Tagen täglich entweder 1200 mg Ibuprofen $(\mathrm{n}=182)$ oder 1500 mg Curcuma domestica (= Synonym von C. longa) $(\mathrm{n}=185)$. Als primäre Zielvariablen wurden die WOMAC-Scores für Schmerzen, Steifheit und Funktionsfähigkeit ermittelt. Weiter wurden unerwünschte Ereignisse (UE) dokumentiert.

Die Baseline-Parameter der beiden Gruppen unterschieden sich nicht. Die erhobenen WOMAC-Scores verbesserten sich in beiden Gruppen in den Wochen 0, 2 und 4 verglichen mit Baseline signifikant. Eine Überprüfung auf Nichtunterlegenheit von Curcuma gegenüber Ibuprofen ergab für den WOMAC-Totalscore sowie für WOMAC-Schmerzen und WOMAC-Funktionsfähigkeit ein signifikant positives Resultat. Curcuma erwies sich in diesen Parametern gegenüber Ibuprofen als nicht unterlegen ( $\mathrm{p}$ $=0,010 ; p=0,018$ bzw. $p=0,010)$. Ein- zig in Bezug aufSteifheit zeigte das Resultat eine nur tendenziell signifikante Nichtunterlegenheit $(p=0,060)$. Die Gesamtzahl der UE war in beiden Gruppen gleich gross. Die Anzahl der Patienten, die über abdominale Schmerzen bzw. Probleme berichteten, war in der Ibuprofen-Gruppe signifikant grösser als in der CurcumaGruppe ( $\mathrm{p}=0,046)$. Die meisten Probanden (96-97\%) waren mit der Behandlung zufrieden, und zwei Drittel der Probanden gaben an, gesamthaft gesehen eine Verbesserung erfahren zu haben.

\section{Zusammenfassung}

Die hier vorgestellten fünf klinischen Studien überprüften die Wirksamkeit von Curcuma-Extrakten bei Arthrose, vor allem bei Kniearthrose sowie rheumatoider Arthritis. Die Gelbwurz-Extrakte wurden entweder gegenüber Placebo oder Diclofenac oder Ibuprofen bzw. gegenüber einer Kombination von Curcuma und einem synthetischen Präparat überprüft. In allen Studien erwies sich der Curcuma-Extrakt als wirksam bzw. zum Teil wirksamer als die Vergleichssubstanz oder die Kombination.

In Bezug auf UE wurden in den Curcuma-Gruppen gesamthaft nicht mehr UE als in den Vergleichsgruppen und signifikant weniger abdominale Beschwerden dokumentiert.

\section{Schlussfolgerung}

Die vorgestellten klinischen Studien dokumentieren die Wirksamkeit und Sicherheit von Curcuma-Extrakten. Damit erweisen sich diese als sinnvolle und sichere Therapie-Alternative zur Behandlung von Arthrosen und insbesondere von Kniearthrosen. 


\section{Literatur}

1 Bachmann C: Neuroprotektive Eigenschaften von Curcumin: Pharmakologische Studien zeigen Wirksamkeit. Schweiz Z Ganzheitsmed 2016;28:259-261.

2 Csaki C, et al: Synergistic chondroprotective effects of curcumin and resveratrol in human articular chondrocytes: inhibition of IL-1betainduced NF-kappaB-mediated inflammation and apoptosis. Arthritis Res Ther 2009;11:R165.

$\checkmark 3$ Chowdhury T, et al: Signal transduction pathways involving p38 MAPK, JNK, NFkappaB and AP-1 influences the response of chondrocytes cultured in agarose constructs to IL-1beta and dynamic compression. Inflamm Res 2008; 57:306-313.
4 Kulkarni RR, et al: Treatment of osteoarthritis with a herbomineral formulation: a doubleblind, placebo-controlled, cross-over study. J Ethnopharmacol 1991;33:91-95.

5 Belcaro G, et al: Efficacy and safety of Meriva ${ }^{\circledR}$, a curcumin-phosphatidylcholine complex, during extended administration in osteoarthritis patients. Altern Med Rev 2010;15:337-344.

6 Chandran B, Goel A: A randomized, pilot study to assess the efficacy and safety of curcumin in patients with active rheumatoid arthritis. Phytother Res 2012;26:1719-1725.
7 Madhu K, Chanda K, Saji MJ: Safety and efficacy of Curcuma longa extract in the treatment of painful knee osteoarthritis: a randomized placebo-controlled trial. Inflammopharmacology 2013;21:129-136.

8 Kuptniratsaikul V, et al: Efficacy and safety of Curcuma domestica extracts compared with ibuprofen in patients with knee osteoarthritis: a multicenter study, Clin Interv Aging 2014;9: 451-458. 